\title{
Die komplizierte Gicht
}

\section{Complications in Gout Arthropathy}

\author{
Autoren \\ Ulrich Illgner ${ }^{1}$, Anna Maier ${ }^{2}$, Ludwig Bause ${ }^{2}$, Henrik Seintsch ${ }^{1}$
}

Institute

1 Orthopädische Privatpraxis Seintsch Illgner, Orthopädisch-unfallchirurgische Privatarztpraxis, Rheumaorthopädie, Technische Orthopädie, Koblenz

2 St. Josef Stift Sendenhorst, Rheumatologisches Kompetenzzentrum Nordwestdeutschland, Klinik für Rheumatologie, Sendenhorst

\section{Schlüsselwörter \\ Gicht, Komplikation, Fokusuche, Infektion}

Key words

gout, complication, focus, infection

Bibliografie

DOI https://doi.org/10.1055/s-0042-110093

Online-Publikation: 19.7.2016

Akt Rheumatol 2017; 42: 551-554

(c) Georg Thieme Verlag KG Stuttgart · New York ISSN 0341-051X

Korrespondenzadresse

Dr. Ulrich Illgner

Orthopädische Privatpraxis Seintsch Illgner Orthopädisch-

unfallchirurgische Privatarztpraxis,

Rheumaorthopädie, Technische Orthopädie

Hohenzollernstraße 64

56068 Koblenz

Tel.: +49/261/988 630, Fax: +49/261/9886 311

ulrich_illgner@web.de

\section{ZUSAMMENFASSUNG}

Ein 57-jähriger Patient wurde aus der internistischen Abteilung einer auswärtigen Klinik übernommen, mit zahlreichen Gichttophi und einer Überwärmung des rechten Knies und des rechten Ellenbogens. Seit 20 Tagen bestand eine probatorische parenterale Antibiotikatherapie. Durch eine sofortige Punktion des rechten Kniegelenks konnte die Diagnose einer bakteriellen Arthritis gestellt werden. Es erfolgte umgehend eine Operation mit Spülung und ausführlichem Debridement unter der Weiterführung der Antibiotikatherapie. Bei Patienten mit Gicht und Verdacht auf eine zusätzliche Infektion muss immer eine vollständige und konsequente Fokussuche und ggf. eine operative Focussanierung durchgeführt werden.

\section{ABSTRACT}

A 57-year-old male was transferred from an external hospital. He had ulcerated massive gout tophi and his right knee and right elbow were hyperthermic. The patient had been treated with parenteral antibiotics for 20 days. The diagnosis of septic arthritis was based on immediate arthrocentesis and synovial fluid analysis of the knee. Subsequent emergency surgery required irrigation and radical debridement. Antibiotic treatment was continued. In patients with gout and signs of infection, a thorough and consistent search for the focus is imperative, as it may be necessary for an orthopaedic surgeon to remove infected synovial tissue.

\section{Anamnese}

Ein 57-jähriger Patient wurde aus der internistischen Abteilung einer auswärtigen Klinik mit einer Überwärmung des rechten Knies, zahlreichen Gichttophi (exulzerierend am rechten Zeigfinger und am rechten Olecranon) übernommen. Aufgrund deutlich erhöhter systemischer Entzündungszeichen und des Fiebers wurde dort seit 20 Tagen eine parenterale Antibiotikatherapie mittels Penicillin/ Sulbactam und seit 14 Tagen additiv mit Clarythromycin durchgeführt, ohne dass eine weitere Focussuche oder eine Gelenkpunktion erfolgt war. In einem unfallchrirugischem Konsil war keine Indikation zur chirurgischen Intervention gestellt worden.
Eigenanamnestisch bestand Nikotinabusus bis vor 4 Jahren und ein dauerhafter Alkoholabusus von 3-4 Flaschen Bier täglich. Eine Medikation mit Allopurinol war zuvor wegen einer Niereninsuffizienz abgesetzt worden. Lokale Steroid-Gabe in Form von Gelenkinjektionen oder systemische Steroidtherapie kamen bisher nicht zum Einsatz; weitere regelmäßige Medikation wurde nicht eingenommen. Etwa 5 Wochen zuvor kam es zum letzten Gichtanfall unter Beteiligung des Mittelgelenks am rechten Zeigefinger, beider Großezehengrundgelenke und der jetzt im Vordergrund stehenden Ellenbogen- und Kniegelenke. Außerdem war vor wenigen Monaten wegen einer gastrointestinalen Blutung eine Laparoto- 
mie zur Umstechung eines Ulkus durchgeführt worden. Weitere Vorerkrankungen mit Hinweisen auf eine Immunsuppression wie Diabetes mellitus bestanden nicht.

\section{Befund}

Klinisch imponierten zunächst die stark von der seit 7 Jahren bekannten Gicht affektierten Ellenbogen, Hände und Finger mit multiplen, teils exulzerierenden Tophi.

Der Patient zeigte einen stark reduzierten Allgemeinzustand und war kaum steh- und nicht gehfähig. Das rechte Ellenbogenund das rechte Kniegelenk zeigten sich überwärmt, gerötet und deutlich geschwollen. Beweglichkeit nach Neutral-Null-Methode für Beugung/Streckung betrug im Knie 100-90-0 und im Ellenbo-

- Tab. 1 Laborwerte des Patienten bei Aufnahme.

\begin{tabular}{|l|l|l|}
\hline & Aufnahme & Referenzwert \\
\hline Leukozyten & $17000 / \mu l$ & $<10000 / \mu l$ \\
\hline CRP & $33 \mathrm{mg} / \mathrm{dl}$ & $<0,5 \mathrm{mg} / \mathrm{dl}$ \\
\hline BSG & $125 \mathrm{~mm} / 1$. Stunde & $6-15 \mathrm{~mm} / 1$. Stunde \\
\hline Kreatinin & $1,46 \mathrm{mg} / \mathrm{dl}$ & $0,70-1,20 \mathrm{mg} / \mathrm{dl}$ \\
\hline eGFR (CKD-EPI-Formel) & $60,2 \mathrm{ml} / \mathrm{min}$ & \\
\hline Procalcitonin & $5,6 \mathrm{mg} / \mathrm{ml}$ & $<2,0 \mathrm{mg} / \mathrm{dl}$ \\
\hline Harnsäure & $7,5 \mathrm{mg} / \mathrm{dl}$ & $3,4-7,0 \mathrm{mg} / \mathrm{dl}$ \\
\hline eGFR: errechnete glomeruläre Filtrationsrate & \\
\hline
\end{tabular}

gen $120-20-0^{\circ}$. Aus mehreren Hautdefekten an der Olecranonspitze entleerten sich teils Uratkristalle, teils trübes Sekret.

\section{Diagnostik}

Parameter der Labordiagnostik bei der Aufnahme sind in der $\triangleright$ Tab. 1 zusammengefasst: Es zeigte sich neben einer Niereninsuffiziens deutlich erhöhte Entzündungszeichen.

Die radiologische Untersuchung zeigte eine Destruktion des Knies ( $\triangleright$ Abb. 1) mit Einbruch der Gelenkflächen. Bei Verdacht auf Empyem wurde unverzüglich das rechte Kniegelenk punktiert. Es entleerte sich putrides Sekret. In der Synoviaanalyse wurden massenhaft Harnsäurekristalle nachgewiesen. Eine Zellzahl konnte aufgrund der Viskosität nicht bestimmt werden. In der Färbung fanden sich in Haufen gelagerte, gram-positive Kokken. Die umgehende interdisziplinäre rheumatologisch-rheumaorthopädische Fallkonferenz beschloss die noch am gleichen Tag erforderliche Operation.

\section{Therapie und Verlauf}

Die Operation begann zunächst mit einer arthroskopischen Spülung. Aufgrund des ausgedehnten Empyems mit Übergreifen auf das angrenzende Weichteilgewebe über die Kapsel hinaus per continuitatem musste auf eine offene radikale Synovialektomie mit Einlagen von mehreren Drainagen gewechselt werden. Auch hier entleerte sich massiv Pus ( $\triangleright$ Abb. 2). Nach 6 Tagen wurde eine geplante Second-Look-Operation mit Lavage des betroffenen Gelenks vorgenommen. 3 Wochen später erfolgte eine erneute Revision bei fortbestehendem Streckdefizit (Beugung/Streckung 90-70-0 ${ }^{\circ}$ ). Hierbei konnte eine vollständige Kniegelenkstreckung erreicht wer-
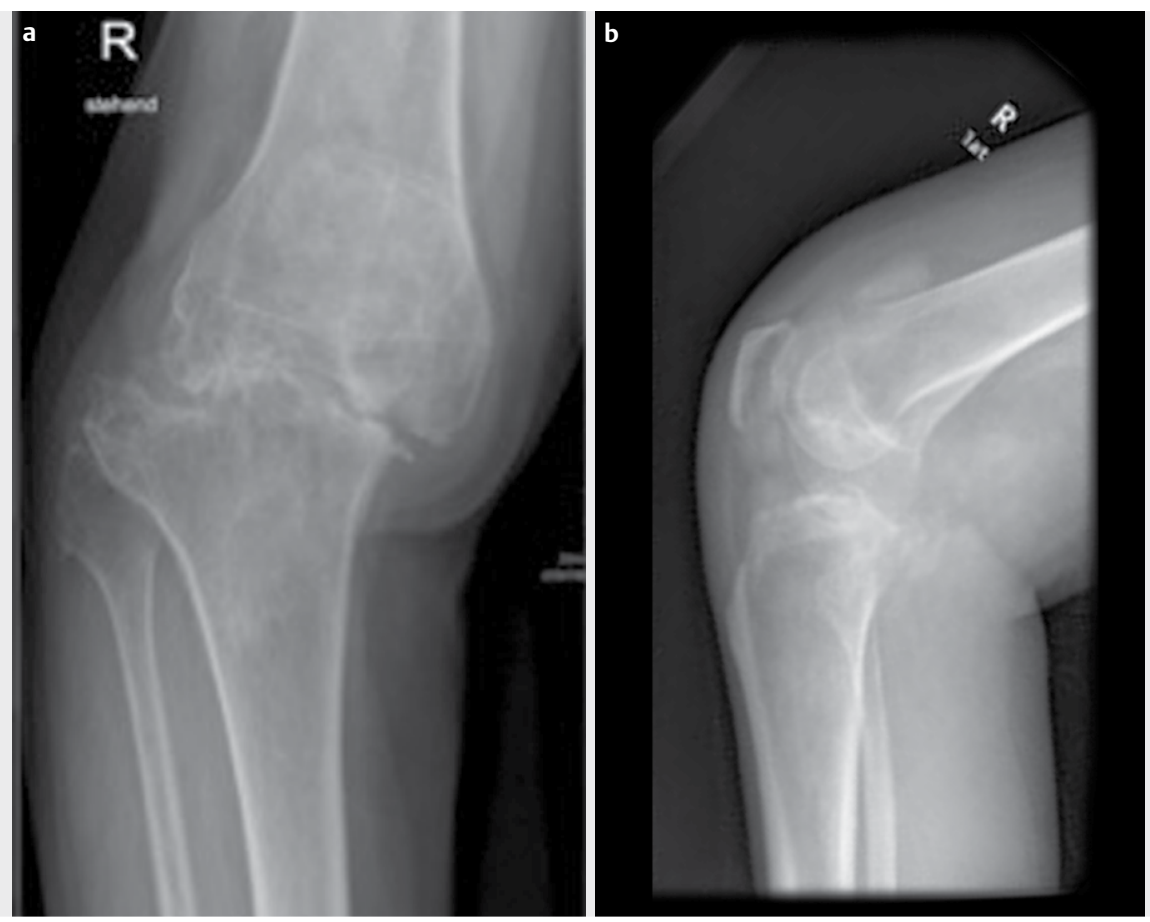

Abb. 1 Röntgenbild zeigt das rechte Knie in 2 Ebenen mit Lyse und Einbruch der Gelenkfläche. 


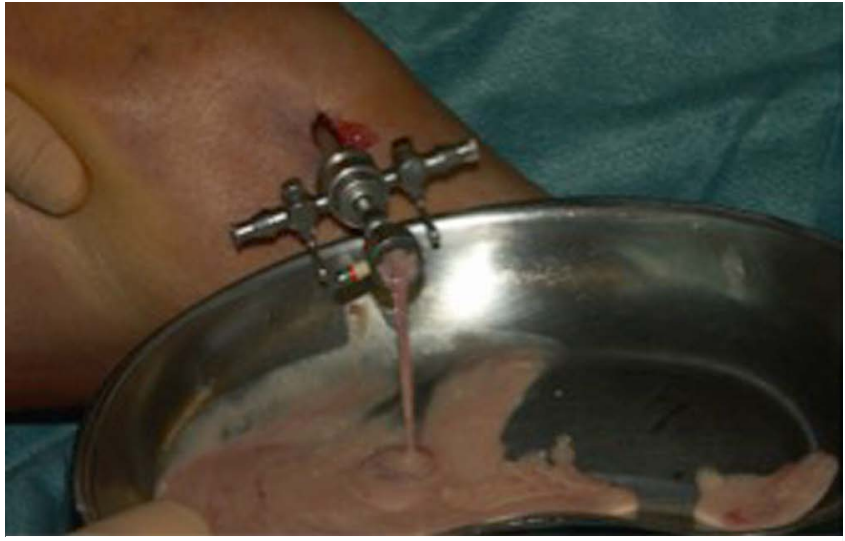

- Abb. 2 Entleerung von putridem Gelenkerguss nach Einbringen des Trokars.

den, supportiv wurde ein Gipstutor mit passiver Durchbewegung des Kniegelenks angepasst. Im Weiteren erfolgte die Mobilisation im Tutor durch die Physiotherapeuten unter schmerzadaptierter Vollbelastung. Am rechten Ellenbogen wurden Spülungen, eine Gipsruhigstellung und lokale Wundbehandlung durchgeführt ( $>$ Abb. 3).

Die mikrobiologische Untersuchung der intraoperativ entnommenen Gewebeproben ergab einen Staphylococcus aureus-Nachweis. Postoperativ wurde die antibiotische Therapie mit den zuvor eingesetzten Substanzen Penicillin/Beta-Lactamase-Inhibitor und Clarythromycin wiederaufgenommen und für 4 Wochen postoperativ fortgesetzt. Die Wirksamkeit entsprach dem Resistogramm.

Bei initialer und postoperativer Anämie wurden Erythrozytenkonzentrate gegeben. Als urikostatische Therapie wurde bei initialem Harnsäure-Wert von 9,6 mg/dl mit Febuxostat $80 \mathrm{mg} /$ Tag begonnen.

\section{Diskussion}

Bei Verdacht auf eine bakterielle Superinfektion der Gelenke kamen in einem externen Krankenhaus über 3 Wochen parenteraler Antibiotika zum Einsatz, die später eine volle Wirksamkeit im Resistogramm zeigten. Diagnostische Punktionen wurden nicht vorgenommen. Aufgrund unzureichendem Ansprechen auf diese Therapie wurde der Patient wurde dann in die hiesige Klinik für Rheumatologie übernommen.

Nach unverzüglicher Punktion des Kniegelenkes wurden in der Synoviadiagnostik des rechten Kniegelenks neben den Harnsäurekristallen auch gram-positive Kokken gefunden. Operativ zeigte sich, dass die Entzündung sich bereits über die Gelenkkapsel hinaus ausgebreitet hatte. Eine Sepsis hätte bei diesem Patienten mit reduziertem Allgemeinzustand und Niereninsuffizienz schnell zu einer lebensbedrohlichen Situation führen können. Die Ganzkörperuntersuchung von Patienten mit schweren Nebenerkrankungen ist immer zwingend erforderlich, auch wenn ein scheinbar offensichtlicher Infektionsherd schon gefunden ist. Möglicherweise war der exulzerierende Tophus am rechten Zeigefinger oder der Hautdefekt am rechten Olecranon die Eintrittsstelle. Anschließend fand eine hämatogene Aussaat in das rechte Knie statt.

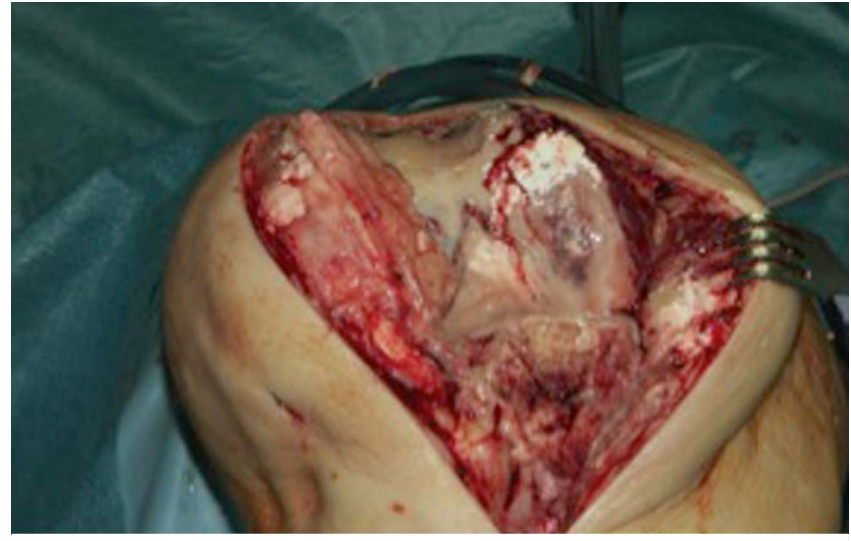

Abb. 3 Intra-operativer Situs mit ausgedehnter entzündlicher Synovialitis und Zerstörung der Gelenkflächen, insbesondere Nekrose der medialen Femurkondyle.

Ohne offensichtliche Eintrittspforten ist eine gründliche Suche nach weiteren Foci, inklusvie der Abdomensonografie und der Echokardiografie zum Ausschluss einer Endokarditis, zwingend und ohne Zeitverlust erforderlich. In einer solchen Situation erscheint eine Punktion aller verdächtigen Gelenke aus Sicht der Autoren unverzichtbar und sollte unverzüglich durchgeführt werden [1]. Die Einsendung einer Nativprobe zum direkten mikrobiologischen Nachweis des Erregers gilt weiter als Goldstandard [2], bei eindeutigen Befunden kann dieses Ergebnis aber nicht abgewartet werden. In unserem Haus wird zusätzlich eine sofortige Gramfärbung durchgeführt. Ist diese positiv, gilt dies als beweisend und es besteht eine Notfall-Operationsindikation. Hier möchten wir auf die AWMF Leitlinien zu bakteriellen Gelenkinfektionen verweisen, auch wenn diese derzeit überarbeitet werden. Das Keimspektrum umfasst auch gram-negative Erreger, z. B. Pseudomas aeruginosa, die schwere Gelenkinfektionen mit nachfolgender Sepsis auslösen können. Bei eindeutigem Nachweis eines Gelenkinfektes stellt die operative Sanierung die Therapie der Wahl dar [1, 3]. Intraoperativ sollten mehrere Gewebeproben entnommen werden. Bei unklaren Befunden empfehlen wir zusätzlich eine histologische Untersuchung, um auf der einen Seite bei Low-grade-Infektionen falsch-negative mikrobiologische Nachweise von wenig virulenten Keimen aufzudecken, auf der anderen Seite aber gerade beim Nachweis von nicht oder wenig pathogenen Keimen oder fehlendem Keimwachstum einen solche Infekt nicht zu übersehen [4-6]. Bei immungeschwächten Patienten können durchaus atypische oder nicht virulente Keime relevante Infektionen verursachen [1,7].

Die Ko-Inzidenz von Gichtarthritis und septischer Arthritis ist schon beschrieben worden, wobei insbesondere auch auf die Schwierigkeit der Differenzialdianose hingewiesen wurde [8-10]. Yu et al. berichten über 30 Fälle von Gicht mit septischer Arthritis [8]. Auch hier war S. aureus der am häufigsten nachgewiesene Keim, mit einer hohen Rate an MRSA von fast $44 \%$. Rupturierte Tophi, wie in dem von uns geschilderten Fall, werden von Yu et al. als Eintrittspforten vermutet [8]. Über 14 Fälle von Gicht- und septischer Arthritis berichten Wenig et al [9]. In dieser Studie wurde meist ein oligoartikulärer Befall mit S. aureus berichtet, ebenso über eine erhöhte Inzidenz von chronischen Nierenerkrankungen 
[9]. Fieber und Beteiligung des Kniegelenkes wird in den meisten Fällen berichtet. Die Ursache des gemeinsamen Auftretens von Gicht und septischer Arthritis blieb in den genannten Studien unklar, ein Zusammenhang mit der Änderung des $\mathrm{pH}$-Wertes durch die Infektion mit sukzessivem Ausfall der Kristalle wird von Wenig vermutet [9].

In spezialisierten Einrichtungen werden die Patienten meist im subakutem Stadium eines Gichtanfalls, wie in diesem Falle, oder mit Komplikationen vorgestellt. In diesem Fall war im Vorfeld aufgrund der Niereninsuffizienz die Medikation mit Allopurinol abgesetzt worden. Nach Sanierung des septischen Focus wurde eine Therapie mit Urikostatikum Febuxostat 80 mg/Tag begonnen.

Nach vollständiger, sicherer Ausheilung der Infektion mit einem Abstand von mindestens 3 Monaten und vorheriger Punktion des Kniegelenkes, um einen persistierenden Infekt auszuschließen, wird eine endoprothetische Versorgung des Kniegelenkes erforderlich sein.

S. aureus wurde als Infektionserreger in dem geschilderten Fall nachgewiesen, was dem zu erwarteten Erregerspektrum in der Literatur entspricht [8-10]. Eine iatrogene septische Arthritis kommt nicht in Betracht, da der Patient in der Vorgeschichte nicht punktiert worden war. Der Alkoholabusus könnte eine das Immunsystem geschwächt haben, beweisen lässt sich das nicht. Es muss immer auch eine MRSA-Infektion in Betracht gezogen werden.

\section{Schlussfolgerung}

Der geschilderte Fall steht im Einklang mit der internationalen Literatur, wobei die Ursache der Ko-Inzidenz von Gicht und septischer Arthritis ungeklärt bleibt. Niereninsuffizienz scheint ein Risikofaktor zu sein. Bei Patienten mit akutem Gichtanfall muss immer an die Möglichkeit einer Infektion gedacht werden. Ein interdisziplinäres Vorgehen ist wichtig und eine gründliche rheumatologische und rheumaorthopädische Focussuche ist zwingend erforderlich. Verdächtige Gelenke sollten unverzüglich punktiert werden. Bei Nachweis von Pus oder positiver Gramfärbung besteht eine sofortige Operationsindikation.
Interessenkonflikt

Die Autoren geben an, dass kein Interessenkonflikt besteht.

\section{Literatur}

[1] AWMF Leitlinie Bakterielle Gelenkinfektionen. Nr. 012/010. Stand der letzten Aktualisierung: 05/2008. @ 2008 Arbeitsgruppe Leitlinien der Dt. Ges. f. Unfallchirurgie. Autorisiert für elektronische Publikation: AWMF online. HTML-Code optimiert: 21.10.2008; 15: 34: 34

[2] Stengel D, Bauwens K. Systematic review and meta-analysis of antibiotic therapy for bone and joint infections. Lancet Infect Dis 2001; 1: $175-188$

[3] Lew DP, Waldvogel FA. Osteomyelitis. Lancet 2004; 364: 369-379

[4] Illgner U, Krenn V, Osada N et al. Histopathologie und Mikrobiologie bei Gelenkinfekten - Erweiterung der Diagnostiksicherheit bei Patienten mit chronischer Polyarthritis. Z Rheumatol 2013; 72 : 709-713 doi:10.1007/s00393-013-1173-2

[5] Krenn V, Morawietz L, Burmester G-R et al. Synovitis score: discrimination between chronic low-grade and high-grade synovitis. Histopathology 2006; 49: 358-364

[6] Kriegsmann J, Hopf T, Jacobs D et al. Molekularpathologische Diagnostik von Gelenkinfektionen. Orthopäde 2009; 38: 531-538

[7] Hettenkofer HJ. Infektiöse (septische) Arthritiden. In: Hettenkofer HJ, (Hrsg). Rheumatologie. 5. Aufl. Thieme; Stuttgart: 2003: 152-155

[8] Yu KH, Luo SF, Liou LB et al. Septic and gouty arthritis - an analysis of 30 cases. Rheumatology 2003; 42: 1062-1066 doi:10.1093/ rheumatology/keg297

[9] Weng CT, Liu MF, Lin LH et al. Rare coexistence of gouty and septic arthritis: a report of 14 cases. Clinical and Experimental Rheumatology 2009; 27: 902-906

[10] Omkar HD, Patel KA, Andersen CR et al. Surgical Procedures Needed to Eradicate Infection in Knee Septic Arthritis. Orthopedics 2016; 39 : $50-54$ 